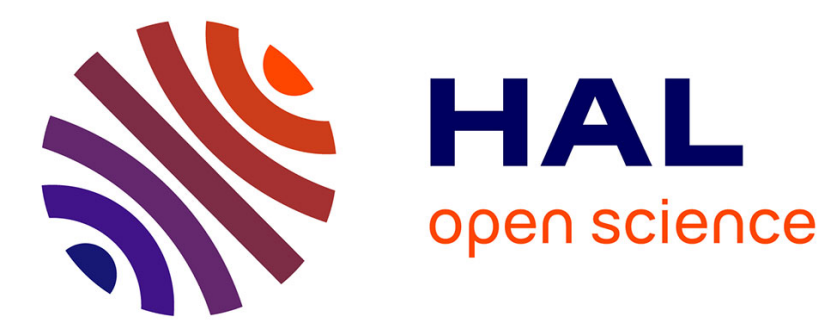

\title{
A Categorical Semantics of Signal Flow Graphs
}

Filippo Bonchi, Pawel Sobociński, Fabio Zanasi

\section{To cite this version:}

Filippo Bonchi, Pawel Sobociński, Fabio Zanasi. A Categorical Semantics of Signal Flow Graphs. CONCUR 2014 - Concurrency Theory - 25th International Conference, Sep 2014, Rome, Italy. hal02134182

\section{HAL Id: hal-02134182 \\ https://hal.science/hal-02134182}

Submitted on 20 May 2019

HAL is a multi-disciplinary open access archive for the deposit and dissemination of scientific research documents, whether they are published or not. The documents may come from teaching and research institutions in France or abroad, or from public or private research centers.
L'archive ouverte pluridisciplinaire HAL, est destinée au dépôt et à la diffusion de documents scientifiques de niveau recherche, publiés ou non, émanant des établissements d'enseignement et de recherche français ou étrangers, des laboratoires publics ou privés. 


\title{
A Categorical Semantics of Signal Flow Graphs
}

\author{
Filippo Bonchi ${ }^{1}$, Paweł Sobociński ${ }^{2}$ and Fabio Zanasi ${ }^{1}$ \\ 1 ENS de Lyon, Université de Lyon, CNRS, INRIA, France \\ 2 ECS, University of Southampton, UK
}

\begin{abstract}
We introduce $\mathbb{I} \mathbb{H}$, a sound and complete graphical theory of vector subspaces over the field of polynomial fractions, with relational composition. The theory is constructed in modular fashion, using Lack's approach to composing PROPs with distributive laws.

We then view string diagrams of $\mathbb{I H}$ as generalised stream circuits by using a formal Laurent series semantics. We characterize the subtheory where circuits adhere to the classical notion of signal flow graphs, and illustrate the use of the graphical calculus on several examples.
\end{abstract}

\section{Introduction}

We introduce a graphical calculus of string diagrams, which we call circuits, consisting of the following constants, sequential ; and parallel $\oplus$ composition.

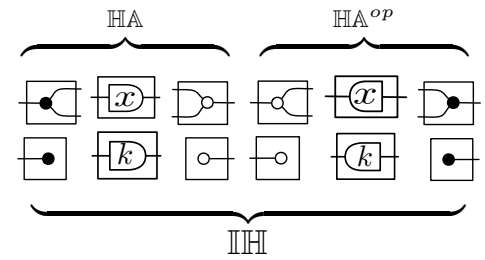

These circuits can be given a stream semantics. The intuition is that wires carry elements of a field $\mathrm{k}$ that enter and exit through boundary ports. In particular, for circuits built from components in the leftmost three columns, which we hereafter refer to as being in $\mathbb{H} \mathbb{A}$, the signal enters from the left and exits from the right boundary. Computation in the circuit proceeds synchronously according to a global "clock", where at each iteration fresh elements are processed from input streams on the left and emitted as elements of output streams on the right.

Intuitively, $\neg$ is a copier, duplicating its input signal; its counit $\bullet$ accepts any signal and discards it, producing no output; $D^{\circ}$ is an adder that takes two inputs and emits their sum, and its unit o- constantly outputs the signal 0; $x$ is a delay, or 1-place buffer that initially holds the 0 value. Finally, $\square$ is an amplifier, multiplying its input by the scalar $k \in \mathrm{k}$. For circuits resulting from the other three columns, $\mathbb{H} \mathbb{A}^{o p}$, the signal flows on the opposite direction: from right to left. The behaviour is symmetric. Formally, the stream semantics of circuits in $\mathbb{H} \mathbb{A}$ and $\mathbb{H} \mathbb{A}^{o p}$ consists of linear transformations of streams. 
Circuits in $\mathbb{I H}$ built out of all the constants above do not, in general, yield functional behaviour. Signals no longer flow in a fixed direction, analogously to how in electrical circuits physical wires are not directed. Indeed, the semantics of circuits in $\mathbb{H} \mathbb{H}$ are not linear maps, but rather subspaces, i.e., linear relations. Passing from functions to relations gives meaning to circuits that contain feedbacks. We must also use an extended notion of streams, Laurent series, typical in algebraic approaches [4] to signal processing - roughly speaking, these streams are allowed to start in the past. Notably, while matrices denoted by circuits in $\mathbb{H} \mathbb{A}$ or $\mathbb{H} \mathbb{A}^{o p}$ only contain streams with a finite number of non-zero values, the subspaces denoted by $\mathbb{I} \mathbb{H}$ are, in general, generated by vectors of streams with infinitely many non-zero values. An example is the Fibonacci circuit (Example 3).

We characterise the stream semantics via both a universal property and an intuitive inductive definition. Furthermore, we provide a sound and complete axiomatization for proving semantic equivalence of circuits.

In order to do that, we consider another canonical semantics for circuits, prior to the stream semantics. We show (Proposition 1) that $\mathbb{H} \mathbb{A}$ is the theory of $\mathrm{k}[x]$-matrices, where $\mathrm{k}[x]$ is the ring of polynomials with coefficients from field $\mathrm{k}$. A modular construction [8] that generalises our earlier contribution [7] allows us to conclude (Theorem 1 ) that $\mathbb{I H}$ is the relational theory of vector subspaces over the field of fractions of $\mathrm{k}[x]$. Then, the passage to the stream semantics simply consists in interpreting polynomials and their fractions as streams. Using again the construction in [8], also this interpretation is given by a universal property.

The study of stream processing circuits has been of significant interest since at least the 1950s [16] and is known as the theory of signal flow graphs (SFG). Traditionally only SFGs that yield functional behaviours on ordinary streams are considered: to ensure this, circuits are restricted so that every feedback loop passes through at least one delay gate. A well-known theorem (see e.g. [14]) states that circuits in this form represent all and only the matrices with entries from $\mathrm{k}\langle x\rangle$, the ring of rational polynomials: those fractions where the constant term in the denominator is non-zero. A novel proof of this result has been recently given by Rutten in [18] by using coinductive and coalgebraic techniques.

Signal flow graphs are first class objects of our theory - they are a certain inductively defined family $\mathbb{S F}$ of circuits in $\mathbb{I H}$. Using its inductive definition, we can give another proof of the aforementioned theorem: $\mathbb{S F}$ is the theory of matrices over $\mathrm{k}\langle x\rangle$ (Theorem 2). The main advantage of our approach is that, by virtue of our full abstraction result, we are able to use graphical equational reasoning on signal flow graphs directly without translations to intermediate linear-algebraic or coalgebraic syntax.

The definition of SFGs given in this paper is very close in spirit to their classical interpretations as graphical structures, which are typically defined in an informal and intuitive fashion, before they are translated to a more formal syntax and abandoned as objects of study. Our main departure from circuit orthodoxy is that we dispense with all notions of input, output and direction of wires. Indeed, guided by the mathematics of circuits, we must consider all of these as derivative notions. By doing so, we are close in spirit to Willems' behavioural approach in 
control theory [22]. Our approach-using string diagrams, which originated in the study of free monoidal categories [20], in order to capture physical systemscan also be considered as a contribution to network theory [2]. Similar ideas lie behind Span(Graph) [12], the algebra of Petri nets with boundaries [10,21] and several algebras of connectors [1]. Independently, Baez and Erbele proposed an equivalent presentation of relational subspaces in their technical report [3], which appeared after the submission of this paper.

There are also close connections to recent work on graphical languages for quantum information. In [7] we used a similar modular construction to characterise the free model of the undirected phase-free version of the ZX-calculus [11]. That construction and the construction of $\mathbb{I} \mathbb{H}$ are both instances of a more general result [8] that we sketch in this paper. Indeed, $\mathbb{I} \mathbb{H}$ can itself be considered as a flavour of directed ZX, albeit with a very different semantic interpretation.

Structure of the paper. In $\S 2$ we recall the required categorical notions. In $\S 3$ we show that $\mathbb{H} \mathbb{A}$ is the graphical theory of $\mathrm{k}[x]$-matrices. In $\S 4$ we give a modular account of $\mathbb{I H}$ and show that it is the theory of relational vector subspaces over the field of fractions of $\mathrm{k}[x]$. In $\S 5$ we focus on the stream semantics and in $\S 6$ we identify an important subclass of $\mathbb{I H}$ : the theory of signal flow graphs.

\section{Background}

$\mathbb{C}[a, b]$ is the set of arrows from $a$ to $b$ in a small category $\mathbb{C}$, composition of $f: a \rightarrow b, g: b \rightarrow c$ is denoted by $f ; g: a \rightarrow c$. For $\mathbb{C}$ symmetric monoidal, $\oplus$ is the monoidal product and $\sigma_{X, Y}: X \oplus Y \rightarrow Y \oplus X$ the symmetry for $X, Y \in \mathbb{C}$. Given $\mathcal{F}: \mathbb{C}_{1} \rightarrow \mathbb{C}_{2}, \mathcal{F}^{o p}: \mathbb{C}_{1}^{o p} \rightarrow \mathbb{C}_{2}^{o p}$ is the induced functor on the opposite categories of $\mathbb{C}_{1}, \mathbb{C}_{2}$. If $\mathbb{C}$ has pullbacks, its span bicategory has the objects of $\mathbb{C}$ as 0 -cells, spans of arrows of $\mathbb{C}$ as 1-cells and span morphisms as 2-cells. We denote with $\operatorname{Span}(\mathbb{C})$ the (ordinary) category obtained by identifying the isomorphic 1-cells and forgetting the 2-cells. Dually, if $\mathbb{C}$ has pushouts, Cospan $(\mathbb{C})$ is the category obtained from the bicategory of cospans.

\section{$2.1 \quad$ PROPs}

A (one sorted) symmetric monoidal theory $(\mathrm{SMT})$ is given by a pair $(\Sigma, E)$ where $\Sigma$ is the signature: a set of operations $o: n \rightarrow m$ with arity $n$ and coarity $m$. The set of $\Sigma$-terms is obtained by composing operations, the identity $i d_{1}: 1 \rightarrow 1$ and symmetry $\sigma_{1,1}: 2 \rightarrow 2$ with ; and $\oplus$ : given $\Sigma$-terms $t: k \rightarrow l, u: l \rightarrow m$, $v: m \rightarrow n$, we construct $\Sigma$-terms $t ; u: k \rightarrow m$ and $t \oplus v: k+n \rightarrow l+n$. The elements of the set $E$ of equations are pairs of $\Sigma$-terms $\left(t, t^{\prime}: k \rightarrow l\right)$.

To study SMTs we use PROPs $[13,15]$ (product and permutation categories). A PROP is a strict symmetric monoidal category with objects natural numbers, where $\oplus$ on objects is addition. Morphisms between PROPs are strict symmetric monoidal functors that act as identity on objects: PROPs and their morphisms form the category PROP. Given an SMT $(\Sigma, E)$, one (freely) obtains a PROP where arrows $k \rightarrow l$ are $\Sigma$-terms $k \rightarrow l$ modulo the laws of symmetric monoidal 
categories and equations $t=t^{\prime}$ where $\left(t, t^{\prime}\right) \in E$. There is a graphical representation of terms as string diagrams (see [20]): we call these diagrams circuits.

For example, let $\left(\Sigma_{M}, E_{M}\right)$ be the SMT of commutative monoids. $\Sigma_{M}$ contains two operation symbols: the multiplication - which we depict as a circuit Do- $: 2 \rightarrow 1-$ and the unit, represented as $0-0 \rightarrow 1$. Graphically, the generation of $\Sigma_{M}$-terms amounts to "tiling" $D^{\circ}$ and $\circ$ together with the circuit $\mp$ (representing $\sigma_{1,1}: 2 \rightarrow 2$ ) and $\square$ (representing $i d_{1}: 1 \rightarrow 1$ ). Equations $E_{M}$ assert associativity (A3), commutativity (A2) and identity (A1).
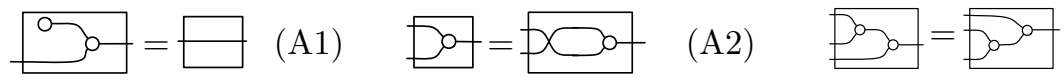

We call $\mathbb{M}^{w}$ the PROP freely generated by $\left(\Sigma_{M}, E_{M}\right) \cdot{ }^{3} \mathrm{~A}$ useful observation is that to give a circuit $c \in \mathbb{M}^{w}[n, m]$ is to give the graph of a function of type $\{0, \ldots, n-1\} \rightarrow\{0, \ldots, m-1\}$. For instance, $\mathfrak{D}^{\circ} \oplus \circ: 2 \rightarrow 2$ describes the function $f:\{0,1\} \rightarrow\{0,1\}$ mapping both elements to 0 . This yields an iso $\mathcal{S}_{\mathbb{M} w}: \mathbb{M}^{w} \rightarrow \mathbb{F}$, where $\mathbb{F}$ is the PROP with arrows $n \rightarrow m$ functions $\{0, \ldots, n-$ $1\} \rightarrow\{0, \ldots, m-1\}$. Intuitively, $\mathbb{F}$ is a "concrete" representation of the theory of commutative monoids and thus we refer to the morphism $\mathcal{S}_{\mathbb{M} w}$ as the denotational semantics of $\mathbb{M}^{w}$.

For later reference, we introduce two more examples of free PROPs. First, let $\mathbb{K}[\mathbb{X}]$ be the PROP freely generated by the signature consisting of $-\not$ for each $p \in \mathrm{k}[x]$ and the following equations, where $p_{1}, p_{2}$ range over $\mathrm{k}[x]$.

$$
+1-\square
$$

$$
-p_{1}-p_{2}-\square
$$

Next, let $\mathbb{C}^{b}$ be the PROP of (black) cocommutative comonoids, freely generated by the signature consisting of circuits $\_, \bullet$ and the following equations.
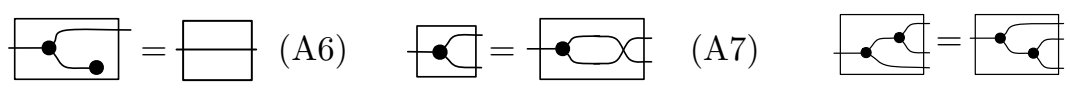

Modulo the white vs. black colouring, the circuits of $\mathbb{C}^{b}$ can be seen as those of $\mathbb{M}^{w}$ "reflected about the $y$-axis". This observation yields that $\mathbb{C}^{b} \cong \mathbb{M}^{w o p}$. More generally, for $\mathbb{T}$ a free PROP, $\mathbb{T}^{o p}$ can be presented by operations and equations which are those of $\mathbb{T}$ reflected about the $y$-axis.

PROPs are also objects of a certain coslice category. First, a PRO is a strict monoidal category with objects the naturals and tensor product on objects addition. Morphisms of PROs are strict identity-on-objects monoidal functors. There is a $\mathrm{PRO}$ of particular interest: the $\mathrm{PRO}$ of permutations $\mathbb{P}$, where $\mathbb{P}[k, l]$ is empty if $k \neq l$ and otherwise consists of the permutations on the set with $k$ elements. Now PROPs are objects of the coslice $\mathbb{P} / \mathbf{P R O}$, where $\mathbf{P R O}$ is the category of PROs. Morphisms of PROPs are thus simply morphisms of PROs that preserve the permutation structure. Working in the coslice is quite intuitive: e.g. $\mathbb{P}$ is the

\footnotetext{
${ }_{3}$ The notation $w$ emphasizes the white colouring of circuits in $\Sigma_{M}$ - later on, we
} will use the black coloring for another copy of the same PROP. 
initial PROP and to compute the coproduct $\mathbb{T}+\mathbb{S}$ in PROP one must identify the permutation structures. When $\mathbb{T}$ and $\mathbb{S}$ are generated by $\left(\Sigma_{C}, E_{C}\right)$ and $\left(\Sigma_{D}, E_{D}\right)$ respectively, it follows that $\mathbb{T}+\mathbb{S}$ is generated by $\left(\Sigma_{C}+\Sigma_{D}, E_{C}+E_{D}\right)$.

\subsection{Composing PROPs}

In [13] Lack showed that co/commutative bialgebras and separable Frobenius algebras stem from different ways of "composing" $\mathbb{C}^{b}$ and $\mathbb{M}^{w}$. Just as small categories are monads in Span(Set), a PROP is a monad in a certain bicategory, and PROPs $\mathbb{T}_{1}$ and $\mathbb{T}_{2}$ can be composed via distributive law $\lambda: \mathbb{T}_{2} ; \mathbb{T}_{1} \rightarrow \mathbb{T}_{1} ; \mathbb{T}_{2}$. A key observation is that the graph of $\lambda$ can be seen as a set of equations. Thus, if $\mathbb{T}_{1}$ and $\mathbb{T}_{2}$ are freely generated PROPs, then so is $\mathbb{T}_{1} ; \mathbb{T}_{2}$.

As an example, we show how composing $\mathbb{C}^{b}$ and $\mathbb{M}^{w}$ yields the PROP of co/commutative bialgebras. First observe that circuits of $\mathbb{C}^{b}$ yield arrows of $\mathbb{F}^{o p}$, because $\mathbb{C}^{b} \cong \mathbb{M}^{w o p} \cong \mathbb{F}^{o p}$. Then a distributive law $\lambda: \mathbb{M}^{w} ; \mathbb{C}^{b} \Rightarrow \mathbb{C}^{b} ; \mathbb{M}^{w}$ has type $\mathbb{F} ; \mathbb{F}^{o p} \Rightarrow \mathbb{F}^{o p} ; \mathbb{F}$, that is, it maps a pair $p \in \mathbb{F}[n, z], q \in \mathbb{F}^{o p}[z, m]$ to a pair $f \in \mathbb{F}^{o p}[n, z], g \in \mathbb{F}[z, m]$. This amounts to saying that $\lambda$ maps cospans $n \stackrel{p}{\rightarrow} z \stackrel{q}{\leftarrow} m$ into spans $n \stackrel{f}{\leftarrow} r \stackrel{g}{\rightarrow} m$ in $\mathbb{F}$. Defining $n \stackrel{f}{\leftarrow} r \stackrel{g}{\rightarrow} m$ as the pullback of $n \stackrel{p}{\rightarrow} z \stackrel{q}{\leftarrow} m$ makes $\lambda$ a distributive law [13]. The resulting PROP $\mathbb{C}^{b} ; \mathbb{M}^{w}$ can be presented by operations - the ones of $\mathbb{C}^{b}+\mathbb{M}^{w}$ - and equations - the ones of $\mathbb{C}^{b}+\mathbb{M}^{w}$ together with those given by the graph of $\lambda$. By definition of $\lambda$, one can read them (in $\mathbb{C}^{b}+\mathbb{M}^{w}$ ) out of the pullback squares in $\mathbb{F}$. For instance:

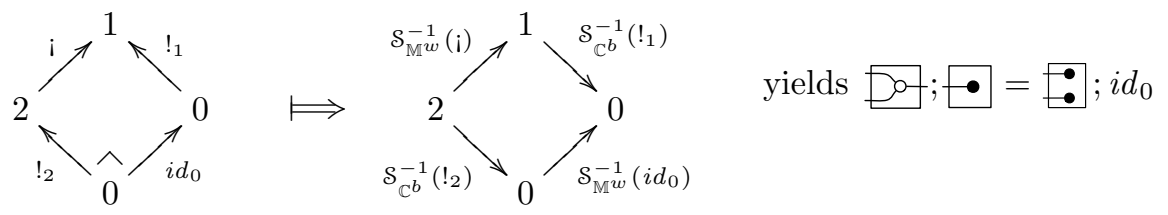

where $\mathrm{i}: 2 \rightarrow 1$ and $!_{n}: 0 \rightarrow n$ are given, respectively, by finality of 1 and initiality of 0 in $\mathbb{F}$, and $\mathcal{S}_{\mathbb{C}^{b}}$ is the isomorphism $\mathbb{C}^{b} \cong \mathbb{F}^{o p}$. In fact, all the equations can be derived from (those of $\mathbb{C}^{b}+\mathbb{M}^{w}$ and) just four pullbacks ( $c f$. [13]) that yield:
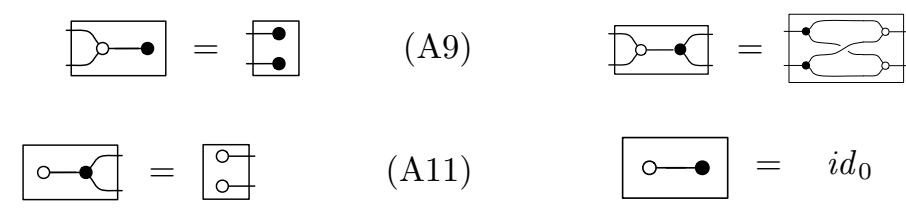

Therefore $\mathbb{C}^{b} ; \mathbb{M}^{w}$ is the free PROP of (black-white) co/commutative bialgebras, obtained as the quotient of $\mathbb{C}^{b}+\mathbb{M}^{w}$ by (A9)-(A12). As another perspective on the same result, one can say that the PROP of co/commutative bialgebras is the theory of $\operatorname{Span}(\mathbb{F}) \cong \mathbb{F}^{o p} ; \mathbb{F}$ and each circuit $c: n \rightarrow m$ of this PROP can be factorised as $c=c_{1} ; c_{2}$, where $c_{1} \in \mathbb{C}^{b}[n, z]$ and $c_{2} \in \mathbb{M}^{w}[z, m]$ for some $z$. 


\section{The Theory of $k[x]$ Matrices}

In this section we introduce the PROP $\mathbb{H} \mathbb{A}$ of $\mathbf{k}[x]$-Hopf Algebras and show that it is isomorphic to the category of matrices over $\mathrm{k}[x]$.

Definition 1. The PROP $\mathbb{H} \mathbb{A}$ is the quotient of $\mathbb{C}^{b}+\mathbb{K}[\mathbb{X}]+\mathbb{M}^{w}$ by the equations (A9), (A11), (A10), (A12) and the following, where $p, p_{1}, p_{2} \in \mathrm{k}[x]$.

$$
\begin{gathered}
D_{-p}=\frac{p}{p-p} \\
O p=\square
\end{gathered}
$$

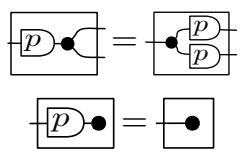

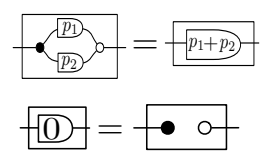

Remark 1. $\mathbb{H} \mathbb{A}$ is a Hopf algebra with antipode $\square=--1$. Indeed it inherits the bialgebra structure of $\mathbb{C}^{b} ; \mathbb{M}^{w}$ and (Hopf) holds by (A4), (A17) and (A18):

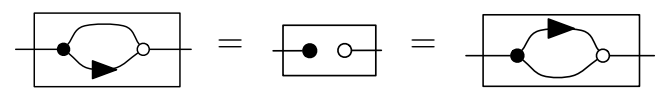

Remark 2. There is an important "operational" intuition associated with circuits in $\mathbb{H} \mathbb{A}$. First, the ports on the left are inputs, the ports on the right are outputs. The circuit constants behave as described in the Introduction, but now wires carry signals which are elements of $\mathrm{k}[x]$, rather than streams.

The theory presented in Definition 1 can be understood in a modular way, in the sense of $\S 2.2$. Reading from left to right, the axioms (A13) and (A14) present a distributive law $\sigma: \mathbb{M}^{w} ; \mathbb{K}[\mathbb{X}] \Rightarrow \mathbb{K}[\mathbb{X}] ; \mathbb{M}^{w}$. Similarly, (A15) and (A16) present a distributive law $\tau: \mathbb{K}[\mathbb{X}] ; \mathbb{C}^{b} \Rightarrow \mathbb{C}^{b} ; \mathbb{K}[\mathbb{X}]$. These laws, together with $\lambda: \mathbb{M}^{w} ; \mathbb{C}^{b} \Rightarrow \mathbb{C}^{b} ; \mathbb{M}^{w}$ which is presented by (A9), (A11), (A10), (A12) (cf. §2.2), yield the composite $\mathbb{C}^{b} ; \mathbb{K}[\mathbb{X}] ; \mathbb{M}^{w}$. We refer to $[8, \S 3]$ for proofs and further details. Now, $\mathbb{H} \mathbb{A}$ is the quotient of $\mathbb{C}^{b} ; \mathbb{K}[\mathbb{X}] ; \mathbb{M}^{w}$ by (A18) and (A17). As a consequence, it inherits the factorisation property of $\mathbb{C}^{b} ; \mathbb{K}[\mathbb{X}] ; \mathbb{M}^{w}$.

Lemma 1 (Factorisation of $\mathbb{H} \mathbb{A}$ ). Any $c \in \mathbb{H} \mathbb{A}[n, m]$ is equal to $s ; r ; t \in$ $\mathbb{H} \mathbb{A}[n, m]$, where $s \in \mathbb{C}^{b}[n, z], r \in \mathbb{K}[\mathbb{X}][z, z]$ and $t \in \mathbb{M}^{w}[z, m]$ for some $z \in \mathbb{N}$.

Lemma 1 fixes a canonical form $s ; r ; t$ for any circuit $c$ of $\mathbb{H} \mathbb{A}$. Furthermore, by (A17), we can assume that any port on the left boundary of $s ; r ; t$ has at most one connection with any port on the right boundary, and by (A4),(A5) we know that any such connection passes through exactly one circuit of shape $p$. We say that a factorised circuit $s ; r ; t$ satisfying this additional requirements is in matrix form. Circuits in matrix form have an intuitive representation as $\mathrm{k}[x]$-matrices, as illustrated in the following example.

Example 1. Consider the circuit $t \in \mathbb{H} \mathbb{A}[3,4]$ and its representation as a $4 \times 3$ matrix $M$ (on the right). For each boundary of $t$, the ports are enumerated from top to bottom, starting from 1 . Then the entry $M_{i, j}$ has value $p \in \mathrm{k}[x]$ if, reading the circuit from the left to the right, one finds a path connecting the $j^{t h}$ port on the left to the $i^{\text {th }}$ port on the right passing through a circuit $\square$, and 0 otherwise.

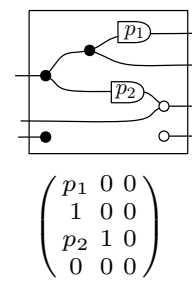


We now make the matrix semantics of circuits in $\mathbb{H} \mathbb{A}$ formal. For this purpose, let Mat $\mathrm{k}[x]$ be the PROP with arrows $n \rightarrow m$ the $m \times n \mathrm{k}[x]$-matrices, where ; is matrix multiplication and $A \oplus B$ is defined as the matrix $\left(\begin{array}{cc}A & 0 \\ 0 & B\end{array}\right)$. The symmetries are the rearrangements of the rows of the identity matrix.

Definition 2. The PROP morphism $\mathcal{S}_{\mathbb{H} \mathbb{A}}: \mathbb{H} \mathbb{A} \rightarrow$ Mat $\mathrm{k}[x]$ is defined inductively:

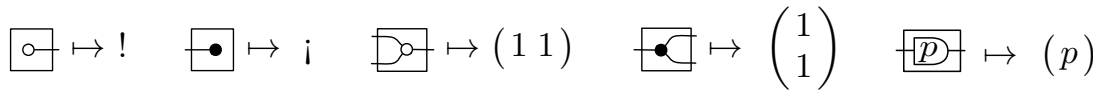

$$
\begin{aligned}
& s \oplus t \mapsto \mathcal{S}_{\mathbb{A B}}(s) \oplus \mathcal{S}_{\mathbb{A B}}(t) \quad s ; t \mapsto \mathcal{S}_{\mathbb{A B}}(s) ; \mathcal{S}_{\mathbb{A B}}(t)
\end{aligned}
$$

where $!: 0 \rightarrow 1$ and $\mathrm{j}: 1 \rightarrow 0$ are given by initiality and finality of 0 in Mat $\mathrm{k}[x]$. It can be checked that $\mathcal{S}_{\mathbb{H A}}$ is well defined, as it respects the equations of $\mathbb{H} \mathbb{A}$.

Proposition 1. $\mathcal{S}_{\mathbb{H} \mathbb{A}}: \mathbb{H} \mathbb{A} \rightarrow$ Mat $\mathrm{k}[x]$ is an isomorphism of PROPs.

Proof. Since the two categories have the same objects, it suffices to prove that $\mathcal{S}_{\mathbb{H A}}$ is full and faithful. For this purpose, observe that, for a circuit $c$ in matrix form, the matrix $\mathcal{S}_{\mathbb{H}}(c)$ can be computed as described in Example 1. Since by Lemma 1 any circuit is equivalent to one of this shape, fullness and faithfulness follows by checking that the encoding of Example 1 is a 1-1 correspondence between matrices and circuits of $\mathbb{H} \mathbb{A}$ in matrix form.

\section{The Theory of Relational $k(x)$ Subspaces}

Let $\mathrm{k}(x)$ denote the field of fractions of $\mathrm{k}[x]$. In this section we introduce the PROP $\mathbb{I} \mathbb{H}$, whose axioms describe the interaction of two $\mathrm{k}[x]$-Hopf algebras, and we show that it is isomorphic to the PROP of $\mathrm{k}(x)$-vector subspaces.

Definition 3. The PROP $\mathbb{H}$ is the quotient of $\mathbb{H} \mathbb{A}+\mathbb{H} \mathbb{A}^{\text {op }}$ by the following equations, where $p$ ranges over $\mathrm{k}[x] \backslash\{0\}$.

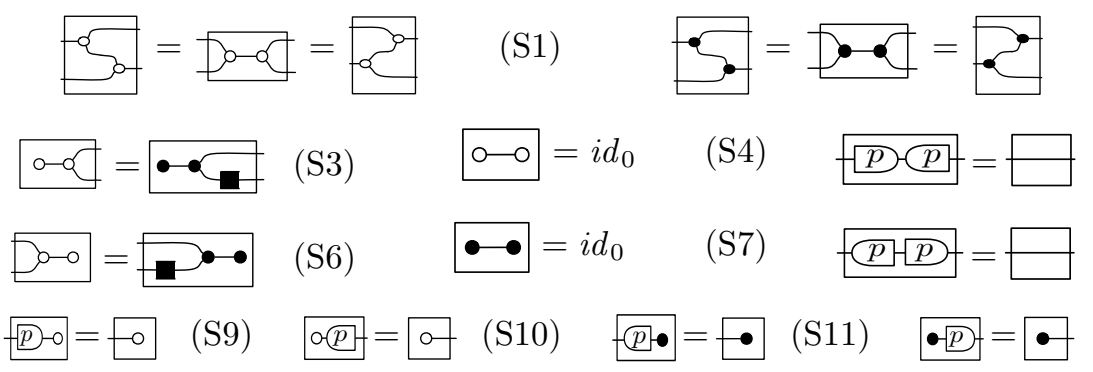

The notation $\square$ indicates both the antipodes $\square$ and $\square$ : indeed, they are equal as circuits of $\mathbb{I} \mathbb{H}$ by virtue of (S5). 
We now consider the task of giving a semantics to circuits of $\mathbb{I H}$. Recall that the semantics of a circuit of $\mathbb{H} \mathbb{A}$ is a matrix, or in other words, a linear transformation. Indeed, as explained in Remark 2, circuits in $\mathbb{H} \mathbb{A}$ can be read from left to right: ports on the left are inputs and ports on the right are outputs.

These traditional mores fail for circuits in $\mathbb{I H}$. Consider $\quad \cdot \cdot 2 \rightarrow 0$ : the component $\bullet$ accepts an arbitrary signal while $D_{\bullet}$ ensures that the signal is equal on the two ports. In other words, the circuit is a "bent identity wire" whose behaviour is relational: the two ports on the left are neither inputs nor outputs in any traditional sense. Indeed, only some circuits of $\mathbb{I H}$ have a functional interpretation. We now introduce the semantic domain of interest for $\mathbb{I} \mathbb{H}$.

Definition 4. Let $\mathbb{S V}_{k(x)}$ be the following PROP:

- arrows $n \rightarrow m$ are subspaces of $\mathrm{k}(x)^{n} \times \mathrm{k}(x)^{m}$ (as a $\mathrm{k}(x)$-vector space).

- composition is relational: for subspaces $G=\left\{(u, v) \mid u \in \mathrm{k}(x)^{n}, v \in \mathrm{k}(x)^{z}\right\}$ and $H=\left\{(v, w) \mid v \in \mathrm{k}(x)^{z}, w \in \mathrm{k}(x)^{m}\right\}$, their composition is the subspace $\{(u, w) \mid \exists v \cdot(u, v) \in G \wedge(v, w) \in H\}$.

- The tensor product $\oplus$ on arrows is given by direct sum of spaces.

- The symmetries $n \rightarrow n$ are induced by bijections of finite sets, $\rho: n \rightarrow n$ is associated with the subspace generated by $\left\{\left(1_{i}, 1_{\rho i}\right)\right\}_{i<n}$ where $1_{k}$ is the binary $n$-vector with 1 at the $k+1$ th coordinate and 0 s elsewhere. For instance $\sigma_{1,1}: 2 \rightarrow 2$ is the subspace generated by $\left\{\left(\left(\begin{array}{l}1 \\ 0\end{array}\right),\left(\begin{array}{l}0 \\ 1\end{array}\right)\right),\left(\left(\begin{array}{l}0 \\ 1\end{array}\right),\left(\begin{array}{l}1 \\ 0\end{array}\right)\right)\right\}$.

Definition 5. Let $\left[v_{1}, \ldots, v_{n}\right]$ denote the space generated by the vectors $v_{1} \ldots v_{n}$. The PROP morphism $\mathcal{S}_{\mathbb{H}}: \mathbb{H} \mathbb{H} \rightarrow \mathbb{S V}_{\mathrm{k}(x)}$ is inductively defined on circuits $c$ of $\mathbb{I} \mathbb{H}$ as follows. For the operations of $\mathbb{H} \mathbb{A}^{4}$ :
$\longmapsto\left[\left(1,\left(\begin{array}{l}1 \\ 1\end{array}\right)\right)\right]$

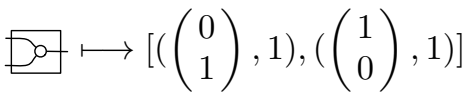
$\bullet \longmapsto[(1,())]$
$\circ \longmapsto[((), 0)]$
$-p-[(1, p)]$

The semantics of an operation $c$ in $\mathbb{H} \mathbb{A}^{o p}$ is symmetric, e.g. $\bullet$ is mapped to $[((), 1)]$. For the composite circuits, we define $c_{1} \oplus c_{2} \mapsto \mathcal{S}_{\mathbb{H} \mathbb{A}}\left(c_{1}\right) \oplus \mathcal{S}_{\mathbb{H} \mathbb{A}}\left(c_{2}\right)$ and $c_{1} ; c_{2} \mapsto \mathcal{S}_{\mathbb{H A}}\left(c_{1}\right) ; \mathcal{S}_{\mathbb{H A}}\left(c_{2}\right)$. The PROP morphism is well-defined since all the equations of $\mathbb{I H}$ are sound w.r.t. $\mathcal{S}_{\mathbb{I H}}$.

The circuit $\bullet$ discussed above is mapped to $\{((p, p),()) \mid p \in \mathrm{k}(x)\} \subseteq$ $\mathrm{k}(x)^{2} \times \mathrm{k}(x)^{0}$. There are similar circuits in $\mathbb{I} \mathbb{H}[2 n, 0]$ for arbitrary $n$.

$$
\epsilon_{0}:=i d_{0} \quad \epsilon_{1}:=\ldots \quad \epsilon_{2}:=\ldots
$$

For instance, $\epsilon_{2}: 4 \rightarrow 0$ has the subspace $\{((p, q, p, q),()) \mid p, q \in \mathrm{k}(x)\}$ as semantics. One can define circuits from 0 to $2 n$ symmetrically, starting from

\footnotetext{
${ }^{4}$ Here and in Definition 6, () denotes the only element of the space with dimension 0.
} 
$\eta_{2}:=\bullet \cdot 0 \rightarrow 2$. As shown in $[8, \S 5]$, the $\eta \mathrm{s}$ and the $\epsilon \mathrm{s}$ form a (self-dual) compact closed structure on the category $\mathbb{I} \mathbb{H}$. This yields a contravariant endofunctor $(\cdot)^{\star}$ on $\mathbb{I} \mathbb{H}(c f .[19$, Rmk 2.1]): for $c: n \rightarrow m$ a circuit, $c^{\star}: m \rightarrow n$ is defined as on the right, where $\bullet^{n} \cdot$ is notation for $\eta_{n}$, $\boldsymbol{D}^{n} \bullet$ for $\epsilon_{n}$ and $n$ for the circuit $i d_{n}$.

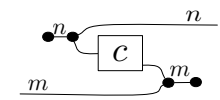

For the sequel, we also fix $n_{\left[p^{n}\right.}$ for the $n$-fold tensor product of $p$. Using the equational theory of $\mathbb{I} \mathbb{H}$, one can show (see $[8, \S 5]$ ) that $c^{\star}$ is just " $c$ reflected

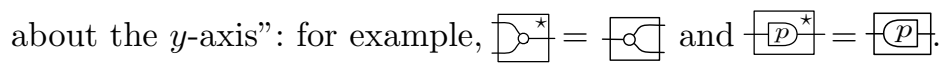

\subsection{Soundness and Completeness of $\mathbb{H} H$ : the Cube Construction}

In this subsection we sketch the proof of the following result, which states that the axioms of $\mathbb{I H}$ (Definition 3) characterise the PROP $\mathbb{S V}_{k(x)}$. The details are in $[8, \S 6-10]$.

Theorem 1. $\mathcal{S}_{\mathbb{H}}: \mathbb{H} \mathbb{H} \rightarrow \mathbb{S V}_{\mathrm{k}(x)}$ is an isomorphism of PROPs.

The proof is interesting in its own right because it is a modular account of the theory of $\mathbb{H} \mathbb{H}$. Its components are summarised by the cube diagram (四) below.

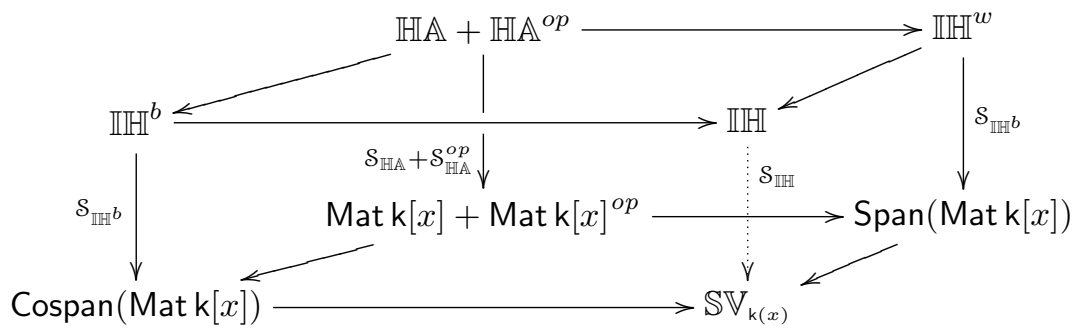

The PROPs that appear in top face of the cube are "syntactic PROPs", i.e., they are freely generated from operations and equations. The PROPs that appear in the bottom face are "semantic PROPs." The vertical morphisms are "denotational semantics" that map terms to their denotations. For example, as we showed in $\S 3, \mathbb{H} \mathbb{A}$ is the theory of matrices with entries from the polynomial ring $\mathrm{k}[x]$, i.e. there is an isomorphism of PROPs $\mathcal{S}_{\mathbb{H} \mathbb{A}}: \mathbb{H} \mathbb{A} \rightarrow$ Mat $\mathrm{k}[x]$.

The theory $\mathbb{I} \mathbb{H}^{w}$ has the presentation of $\mathbb{I} \mathbb{H}$ (Definition 3) but with the two leftmost axioms below replacing (S7), (S8), (S11) and (S12). Dually, $\mathbb{H}^{b}$ is $\mathbb{I} \mathbb{H}$ without (S4), (S5), (S9) and (S10), and the addition of the two rightmost axioms below (the four of them are derivable in $\mathbb{I} \mathbb{H}$, see [8]).

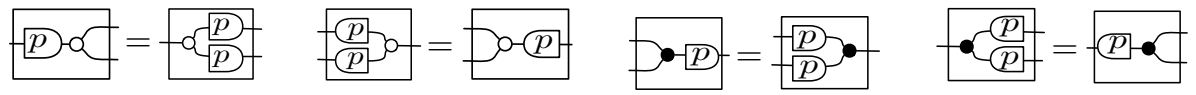

In fact, $\mathbb{I} \mathbb{H}^{w}$ and $\mathbb{I H}^{b}$ are the theories of (i.e. there are isos $\mathcal{S}_{\mathbb{H} \mathbb{H}^{b}}$ and $\mathcal{S}_{\mathbb{I} \mathbb{H}^{w}}$ ) Span(Matk k $[x]$ ) and Cospan(Matk $[x])$, respectively. First we focus on $\mathbb{H}^{w}$ and 
Span(Mat $\mathrm{k}[x])$. Note that pullbacks in Mat $\mathrm{k}[x]$ exist and are computed as in the category of sets, since $\mathrm{k}[x]$ is a principal ideal domain (PID).

The pullback construction gives a distributive law of PROPs in the sense of Lack [13] and, as we explained in $§ 2.2$, pullbacks can be understood as "adding new equations" to the theory $\mathbb{H} \mathbb{A}+\mathbb{H} \mathbb{A}^{o p}$. Indeed, for each of the axioms of $\mathbb{I} \mathbb{H}^{w}$ there is a corresponding "witnessing" pullback in Mat $\mathrm{k}[x]$ : this argument confirms the soundness of the theory of $\mathbb{I} \mathbb{H}^{w}$ for Span(Matk $\left.[x]\right)$. The task of demonstrating the completeness of the axioms is more subtle: one has to prove that the axioms are sufficient for deriving any equation that arises from a pullback in Matk $[x]$. The proof amounts to showing that classical linear algebraic manipulations on matrices that are performed when calculating the kernel of a linear transformation can be mimicked graphically in $\mathbb{H}^{w}$. Due to space constraints, we refer to our technical report $[8, \S 6]$ for the details.

Having constructed the isomorphism between $\mathbb{I} \mathbb{H}^{w}$ and Span(Mat $\mathrm{k}[x]$ ), we can use the fact that the transpose operation on matrices induces a duality in Mat $\mathrm{k}[x]$ to yield the isomorphism between $\mathbb{I H}^{b}$ and Cospan(Mat $\mathrm{k}[x]$ ).

Now let us again focus on the top face of (四). It is a pushout diagram in PROP: as only "syntactic" (freely generated) PROPs are involved, this simply amounts to saying that the equational theory of $\mathbb{I H}$ can be presented as the union of the equational theories of $\mathbb{I} \mathbb{H}^{w}$ and $\mathbb{I} \mathbb{H}^{b}$. An appealing consequence of this construction is that $\mathbb{I H}$ inherits the factorisation properties of both composed PROPs $\mathbb{I} \mathbb{H}^{w}$ and $\mathbb{H}^{b}$, that is, any circuit of $\mathbb{I} \mathbb{H}$ can be put (via the equational theory of $\mathbb{H} \mathbb{H}$ ) in the form of a span or a cospan of circuits of $\mathbb{H} \mathbb{A}$.

The final ingredient in the proof is showing that the bottom face of (四) is also a pushout diagram in PROP. We would like to draw the reader's attention to the remarkable fact that subspaces over the field of fractions $\mathrm{k}(x)$ of $\mathrm{k}[x]$ arise from pushing out spans and cospans of $\mathrm{k}[x]$-matrices along zig-zags of $\mathrm{k}[x]$ matrices. This fact holds for an arbitrary PID and its field of fractions: the elementary proof of this result can be found in $[8, \S 9]$.

Now, we have a commutative cube in which the top and bottom face are pushouts, and the three rear vertical morphisms are isomorphisms. The universal property of pushouts now ensures that the unique morphism $\mathcal{S}_{\mathbb{H}}: \mathbb{I} \mathbb{H} \rightarrow \mathbb{S V}_{k(x)}$ is an isomorphism: it is easy to verify that it is the morphism of Definition 5 .

\section{Stream Semantics}

With simple extensions of the semantics morphisms, we can interpret circuits of $\mathbb{H} \mathbb{A}$ and $\mathbb{I H}$ in terms of streams. First we need to recall some useful notions.

A formal Laurent series (fls) is a function $\sigma: \mathbb{Z} \rightarrow \mathrm{k}$ for which there exists $i \in \mathbb{Z}$ such that $\sigma(j)=0$ for all $j<i$. The degree of $\sigma$ is the smallest $d \in \mathbb{Z}$ such that $\sigma(d) \neq 0$. We write $\sigma$ as $\ldots, \sigma(-1), \sigma(0), \sigma(1), \ldots$ with position 0 underlined, or as formal sum $\sum_{i=d}^{\infty} \sigma(i) x^{i}$. With the latter notation, we define the sum and product of two fls $\sigma=\sum_{i=d}^{\infty} \sigma(i) x^{i}$ and $\tau=\sum_{i=e}^{\infty} \tau(i) x^{i}$ as

$$
\sigma+\tau=\sum_{i=\min (d, e)}^{\infty}(\sigma(i)+\tau(i)) x^{i} \quad \sigma \cdot \tau=\sum_{i=d+e}^{\infty}\left(\sum_{k+j=i} \sigma(j) \cdot \tau(k)\right) x^{i}
$$


The units for + and $\cdot$ are $\ldots 0, \underline{0}, 0 \ldots$ and $\ldots 0, \underline{1}, 0 \ldots$ Fls form a field $\mathrm{k}((x))$, where the inverse $\sigma^{-1}$ of fls $\sigma$ with degree $d$ is given as follows.

$$
\sigma^{-1}(i)= \begin{cases}0 & \text { if } i<-d \\ \sigma(d)^{-1} & \text { if } i=-d \\ \frac{\sum_{i=1}^{n}\left(\sigma(d+i) \cdot \sigma^{-1}(-d+n-i)\right)}{-\sigma(d)} & \text { if } i=-s+n \text { for } n>0\end{cases}
$$

A formal power series (fps) is a fls with degree $d \geq 0$. By (1), fps are closed under + and $\cdot$, but not under inverse: it is immediate by (2) that $\sigma^{-1}$ is a fps iff $\sigma$ has degree $d=0$. Therefore fps form a ring which we denote by $\mathrm{k}[[x]]$.

We will refer to both fps and fls as streams. Indeed, fls can be thought of as sequences with an infinite future, but a finite past. Just as a polynomial $p$ can be seen as a fraction $\frac{p}{1}$, an fps $\sigma$ can be interpreted as the fls $\ldots, 0, \sigma(0), \sigma(1), \sigma(2), \ldots$ A polynomial $p_{0}+p_{1} x+\cdots+p_{n} x^{n}$ can also be regarded as the fps $\sum_{i=0}^{\infty} p_{i} x^{i}$ with $p_{i}=0$ for all $i>n$. Similarly, fractions can be regarded as fls: we define $\tilde{r}: \mathrm{k}(x) \rightarrow \mathrm{k}((x))$ as the unique field morphism mapping $k \in \mathrm{k}$ into the stream $\ldots 0, \underline{k}, 0 \ldots$ and the indeterminate $x$ into $\ldots, 0, \underline{0}, 1,0, \ldots$ Differently from polynomials, fractions can denote streams with possibly infinitely many non-zero values. $\mathrm{k}[[x]] \stackrel{\mathrm{C}}{\longrightarrow} \mathrm{k}((x))$ For instance, (1) and (2) imply that $\frac{x}{1-x-x^{2}}$ is the Fibonacci series $\ldots, 0, \underline{0}, 1,1,2,3, \ldots$ Moreover, while polynomials can be interpreted as fps, fractions need the full generality of fls: $\frac{1}{x}$ denotes $\ldots 0,0,1, \underline{0}, 0, \ldots$

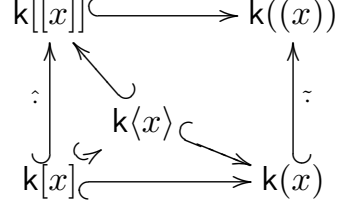

These are all ring morphisms and are illustrated by the commutative diagram on the right. At the center there is $\mathrm{k}\langle x\rangle$, the ring of rationals, i.e, fractions of polynomials $\frac{k_{0}+k_{1} x+k_{2} x^{2} \cdots+k_{n} x^{n}}{l_{0}+l_{1} x+l_{2} x^{2} \cdots+l_{n} x^{n}}$ where $l_{0} \neq 0$. Differently from fractions, rationals denote only fps - in other words, bona fide streams that do not start "in the past". Indeed, since $l_{0} \neq 0$, the inverse of $l_{0}+l_{1} x+l_{2} x^{2} \cdots+l_{n} x^{n}$ is, by (2), a fps. The streams denoted by $k\langle x\rangle$ are well known in literature under the name of rational streams [6]. Hereafter, we will use polynomials and fractions to denote the corresponding streams. Moreover, Mat $\mathrm{k}[[x]]$ and Mat $\mathrm{k}\langle x\rangle$ denote the PROPs of matrices over $\mathrm{k}[[x]]$ and $\mathrm{k}\langle x\rangle$ defined analogously to Mat $\mathrm{k}[x]$. Similarly, $\mathbb{S V}_{\mathbf{k}((x))}$ is the PROP of $\mathrm{k}((x))$-vector subspaces defined like $\mathbb{S V}_{\mathrm{k}(x)}$.

\subsection{A stream semantics of $\mathbb{H} \mathbb{A}$}

The semantics $\mathcal{S}_{\mathbb{H A}}: \mathbb{H} \mathbb{A} \rightarrow$ Matk $[x]$ of Definition 2 allows us to regard the circuits in $\mathbb{H} \mathbb{A}$ as stream transformers. Indeed, the interpretation of a polynomial in $\mathrm{k}[x]$ as a fps in $\mathrm{k}[[x]]$ can be pointwise extended to a faithful PROP morphism $\hat{\therefore}$ Mat $\mathrm{k}[x] \rightarrow$ Mat $\mathrm{k}[[x]]$. By taking $\llbracket \cdot \rrbracket_{\mathbb{H} \mathbb{A}}=\mathcal{S}_{\mathbb{H A}} ; \hat{;}$, the semantics $\llbracket c \rrbracket_{\mathbb{H A}}$ of a circuit $c \in \mathbb{H} \mathbb{A}[n, m]$ consists of a linear map of type $\mathrm{k}[[x]]^{n} \rightarrow \mathrm{k}[[x]]^{m}$.

Remark 3. Recall the operational intuition for circuits in $\mathbb{H} \mathbb{A}$ given in Remark 2. This intuition extends to the stream semantics, but rather than carrying elements of $\mathrm{k}[x]$ along the wires, the circuits now carry individual elements of a k-stream, 
processing one after the other. Inputs arrive on the left and outputs are emitted on the right. For instance, $\llbracket\left[x \rrbracket_{\mathbb{H A}}=(x)\right.$ maps every stream $\sigma \in \mathbf{k}[[x]]$ into the stream $\sigma \cdot x$ which, by (1), is just $\underline{0}, \sigma(0), \sigma(1), \sigma(2), \ldots$ Thus $-x$ behaves as a delay. Instead, for $k \in \mathrm{k}, \llbracket \mathbb{k} \mathbb{H \mathbb { A }}=(k)$ maps $\sigma$ into $\sigma \cdot k=k \sigma(0), k \sigma(1), k \sigma(2), \ldots$ Therefore $-k$ acts as an amplifier. Also $D$ - behaves as an adder and its unit $\circ$ as the constant stream $\underline{0}, 0,0 \ldots$ The comultiplication $\rightarrow$ acts as copier and its counit $\rightarrow$ as the trivial transformer taking any stream in input and giving no output.

One can readily check that this interpretation coincides with the semantics given in $[18, \S 4.1]$. Our approach has the advantage of making the circuits representation formal and allowing for equational reasoning, as shown for instance in Example 2 below. Indeed, since $\llbracket \cdot \|_{\mathbb{H} \mathbb{A}}: \mathbb{H} \mathbb{A} \rightarrow$ Mat $\mathrm{k}[[x]]$ is faithful, the axiomatization of $\mathbb{H} \mathbb{A}$ is sound and complete.

Example 2. Consider the following derivation in the equational theory of $\mathbb{H} \mathbb{A}$, where (A15) is used at each step.

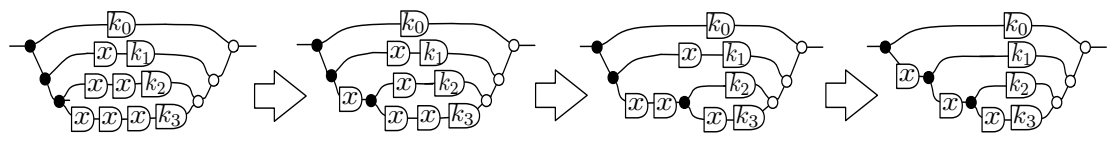

Using the stream interpretation of Remark 3, the circuits above are readily seen to implement the polynomial stream function $f: \sigma \mapsto \sigma \cdot p$ where $p=$ $\underline{k_{0}}, k_{1}, k_{2}, k_{3}, 0 \ldots$ Then (3) yields a procedure that reduces the total number of delays $-x$ - appearing in the circuit ( $c f$. [18, Prop. 4.12]). The equational theory of $\mathbb{H} \mathbb{A}$ allows us to verify that the circuits in (3) really implement $f$ :

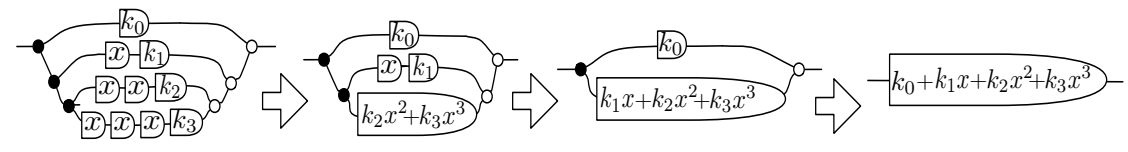

By iteratively applying (A5) and (A17), the circuit is show to be equal to $D$, which clearly has the $1 \times 1$ matrix $(p)$ as semantics and thus implements $f$. Reading (4) in the converse direction, it yields a decomposition of $D$ into an equivalent circuit with only "basic gates": amplifiers $-[$ (for $k \in \mathrm{k}$ ) and delays $-x$ - in fact, such a decomposition is possible for arbitrary circuits of $\mathbb{H} \mathbb{A}$. 


\subsection{A stream semantics of $\mathbb{I H}$}

In order to give the stream interpretation of $\mathbb{H} \mathbb{H}$, we construct the following cube, the topmost face of which coincides with the bottom face of (四).

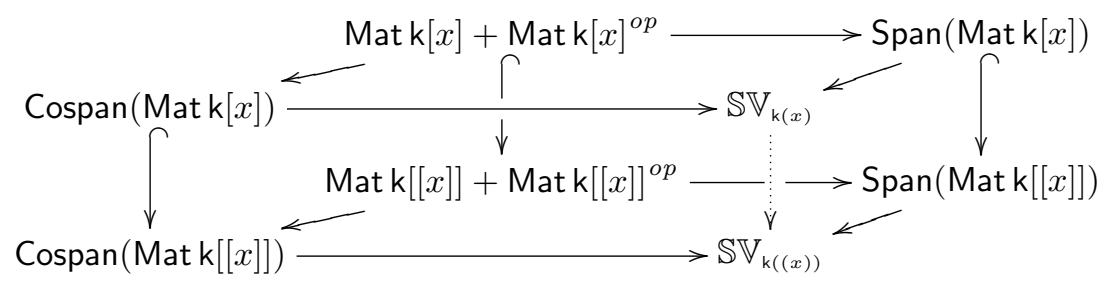

The bottom face commutes and is a pushout for the same reasons as the top face $(c f . \S 4.1)$, because $\mathrm{k}[[x]]$ is a PID and $\mathrm{k}((x))$ is its field of fractions. The rear map is $\hat{\imath}+\hat{\iota}^{o p}:$ Mat $\mathrm{k}[x]+\operatorname{Mat} \mathrm{k}[x]^{o p} \rightarrow \operatorname{Mat} \mathrm{k}[[x]]+$ Mat $\mathrm{k}[[x]]^{o p}$. Since $\hat{\text { ? }}$ preserves pullbacks, we can define the righmost vertical morphism as mapping a span $n \stackrel{V}{\longleftarrow} z \stackrel{W}{\longrightarrow} m$ into $n \stackrel{\hat{V}}{\longleftarrow} z \stackrel{\hat{W}}{\longrightarrow} m$. The leftmost vertical map is defined analogously.

One can readily check that all these morphisms are faithful and that the rear faces commute. Since the top face is a pushout, the universal property induces the faithful morphism [r]: $\mathbb{S V}_{\mathrm{k}(x)} \rightarrow \mathbb{S V}_{\mathrm{k}((x))}$. This can be concretely defined by observing that $\because: \mathrm{k}(x) \rightarrow \mathrm{k}((x))$ can be pointwise extended to matrices and sets of vectors. For a subspace $H$ in $\mathbb{S V}_{k(x)},[\tilde{H}]$ is the space in $\mathbb{S V}_{k((x))}$ generated by the set of vectors $\tilde{H}$. Note that the composition of $\mathcal{S}_{\mathbb{H} \mathbb{H}}$ (Definition 5) with [־], that we call the stream semantics of $\mathbb{H} \mathbb{H}$, is also induced by the universal property of the topmost face of (四).

Definition 6. The stream semantics of $\mathbb{I} \mathbb{H}$ is the morphism $\llbracket \cdot \rrbracket_{\mathbb{H}}: \mathbb{I} \mathbb{H} \rightarrow \mathbb{S V}_{\mathrm{k}((x))}$ defined as $\mathcal{S}_{\mathbb{H}} ;[\because]$. It can be presented as follows. For the operations of $\mathbb{H} \mathbb{A}$ :

$$
\begin{aligned}
& \bullet \longmapsto\left\{\left(\sigma,\left(\begin{array}{c}
\sigma \\
\sigma
\end{array}\right)\right) \mid \sigma \in \mathrm{k}((x))\right\} \quad \overline{D^{\circ}} \longmapsto\left\{\left(\left(\begin{array}{c}
\sigma \\
\tau
\end{array}\right), \sigma+\tau\right) \mid \sigma, \tau \in \mathrm{k}((x))\right\}
\end{aligned}
$$

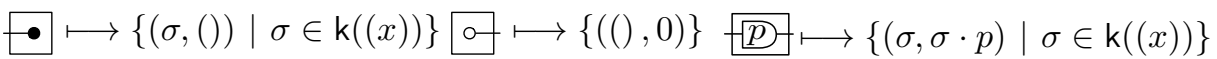

where 0 and $p$ denote streams. The semantics of an operation $c$ of $\mathbb{H} \mathbb{A}^{\text {op }}$ is the reverse relations of $\llbracket c^{\star} \rrbracket_{\mathbb{H}}$. For composite circuits, we let $c_{1} \oplus c_{2} \mapsto \llbracket c_{1} \rrbracket_{\mathbb{H A}} \oplus \llbracket c_{2} \rrbracket_{\mathbb{H A}}$ and $c_{1} ; c_{2} \mapsto \llbracket c_{1} \rrbracket_{\mathbb{H A}} ; \llbracket c_{2} \rrbracket_{\mathbb{H A}}$.

Since $[\because]$ is faithful, by Theorem 1 , also $\llbracket \cdot \rrbracket_{\mathbb{H}}$ is faithful.

Corollary 1 (Completeness). For all $c_{1}, c_{2} \in \mathbb{I H}, c_{1}=c_{2}$ iff $\llbracket c_{1} \rrbracket_{\mathbb{H} \mathbb{H}}=\llbracket c_{2} \rrbracket_{\mathbb{H} \mathbb{H}}$.

\section{The Theory of Signal Flow Graphs}

In this section we introduce an inductively defined class of circuits $\mathbb{S F}$ of $\mathbb{I} \mathbb{H}$ that we call signal flow graphs and show that it is the theory of $\mathrm{k}\langle x\rangle$-matrices. The 
definition is close in spirit to the classical variations found in the literature sans inputs, outputs and directions of wires.

We start with a motivating example of a circuit not in $\mathbb{H} \mathbb{A}$ that nevertheless gives functional behaviour on $\mathrm{k}[[x]]$.

Example 3. The rational $\frac{x}{1-x-x^{2}}$ denoting the Fibonacci sequence can be succintly represented in $\mathbb{I H}$ as the circuit $-x-\sqrt{1-x-x^{2}}$. Indeed, composing the semantics $[(1, x)]$ of $-x$ with the semantics $\left[\left(1-x-x^{2}, 1\right)\right]$ of $-1-x-x^{2}-$ yields the $\mathrm{k}((x))$-subspace $\left[\left(1, \frac{x}{1-x-x^{2}}\right)\right]$. The derivation in the equational theory of $\mathbb{H} \mathbb{H}$ below shows how we can "implement" the Fibonacci circuit.

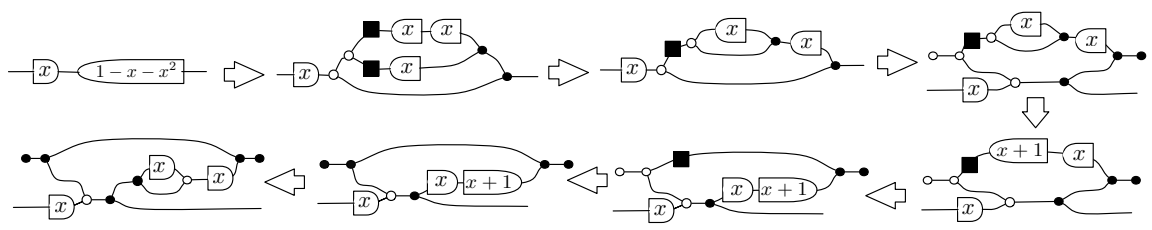

First $1-x-x^{2}$ is decomposed (using (A17) from $\mathbb{H A}^{o p}$ ) and then the circuit is "deformed" in a suitable way by essentially using the Frobenius axioms (S2)-(S1) and the compact closed structure of $\mathbb{I H}$. The resulting circuit exhibits a feedback structure. Indeed, using the intuitive operational descriptions of Remark 3 and the behaviour of $\bullet, \bullet$ as "bent identity wires" that merely forward signals from one port to the other, the operational behaviour of the final circuit in the derivation can be "read off" the final circuit, with inputs entering on the left and outputs emitted on the right. In particular, the reader will verify that inputing the stream $\ldots, 0, \underline{1}, 0, \ldots$ yields the Fibonacci sequence as output. Note that the notions of "input", "output" and directionality of wires are entirely derivative.

The Fibonacci circuit belongs to the class of circuits $\mathbb{S F}$. To define it, we first introduce a particular trace structure $[20, \S 5.1]$ on $\mathbb{I} \mathbb{H}$. It is not the canonical trace induced by the compact closed structure, but rather a "guarded" version.

Definition 7. For $n, m, z \in \mathbb{N}, c \in \mathbb{I} \mathbb{H}[z+n, z+m]$, the $z$-feedback $\operatorname{Tr}^{z}(c) \in$ $\mathbb{I} \mathbb{H}[n, m]$ is the circuit below, for which we use the indicated shorthand notation:

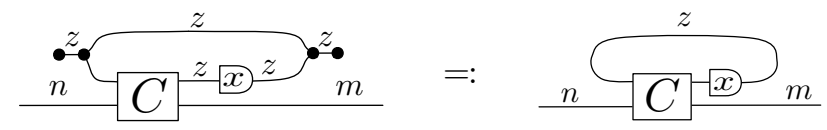

It can be verified that $\operatorname{Tr}^{(\cdot)}$ actually defines a trace on $\mathbb{I} \mathbb{H}$. We have now all the ingredients to define the theory of signal flow graphs.

Definition 8. Let $\mathbb{S F}$ be the following, inductively defined set of circuits.

- If $c \in \mathbb{H} \mathbb{A}[n, m]$ then $c$ is in $\mathbb{S F}$.

- If $c \in \mathbb{S F}[z+n, z+m]$, then $\operatorname{Tr}^{z}(c) \in \mathbb{I} \mathbb{H}[n, m]$ is in $\mathbb{S F}$. 
Circuits in $\mathbb{S F}$ inherit the equational theory of $\mathbb{I} \mathbb{H}$, that is, we say that $c=c^{\prime}$ as circuits in $\mathbb{S F}$ exactly when $c=c^{\prime}$ in $\mathbb{I H}$.

One can check that $\mathbb{S F}$ is a sub PROP of $\mathbb{I} \mathbb{H}$, namely the smallest one containing $\mathbb{H} \mathbb{A}$ and closed under the trace. This is essential for proving that $\mathbb{S F}$ is the theory of $\mathrm{k}\langle x\rangle$-matrices.

Theorem 2. There is an isomorphism of PROPs between $\mathbb{S F}$ and Mat $\mathrm{k}\langle x\rangle$.

Proof. Hereafter, we sketch one direction of the isomorphism, namely from Mat $\mathrm{k}\langle x\rangle$ to $\mathbb{S F}$. The main insight is that any $(1 \times 1$ matrix with a) rational of the form $1 /(k+x p)$, with $k \neq 0$ and $p \in \mathrm{k}[x]$, corresponds to a circuit in $\mathbb{S F}$, as witnessed by the following derivation in $\mathbb{I} \mathbb{H}$ :

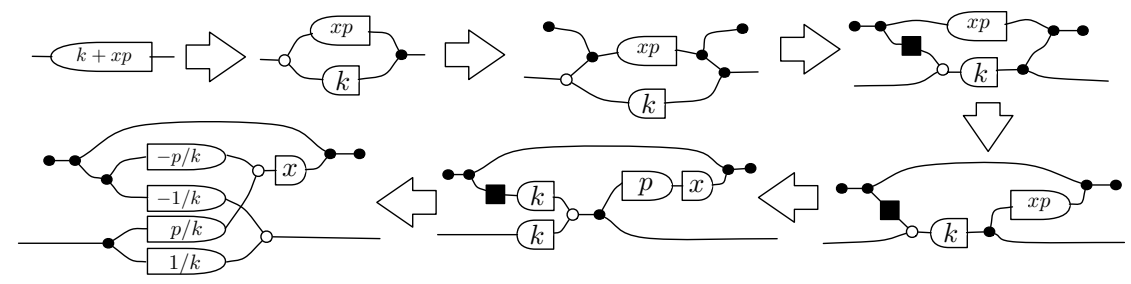

Then, every matrix in Mat $k\langle x\rangle$ is obtained by composing these circuits with those in $\mathbb{H} \mathbb{A}$. Such composition is still in $\mathbb{S F}$, since $\mathbb{S F}$ is a PROP.

\section{Conclusions}

We introduced $\mathbb{H} \mathbb{H}$, a graphical calculus of streams transformers equipped with a compositional semantics in terms of subspaces and a sound and complete axiomatisation. We have identified a fragment of $\mathbb{I} \mathbb{H}$ characterising signal flow graphs, which are functional streams transformers. To best of our knowledge, the axioms of $\mathbb{I} \mathbb{H}$ provides the first sound and complete axiomatisation of signal flow graphs seen as syntactic entities. Indeed, previous results either restrict the class of systems (for instance [17] only considers the "closed" ones) or exploit an intermediate (co)algebraic syntax (see e.g. [5] and the references therein).

However, our interest in $\mathbb{I H}$ is not restricted to signal flow graphs: the circuits of $\mathbb{I H}$ are streams transformers which are, in general, relational rather than functional. Such relational behaviour emerges naturally when studying different sorts of networks $[1,2,4,9-12,21]$.

Acknowledgements The first and the third author acknowledge support from the ANR project 12IS02001 PACE.

\section{References}

1. F. Arbab. Reo: a channel-based coordination model for component composition. Mathematical Structures in Computer Science, 14:329-366, 62004. 
2. J. C. Baez. Network theory. http://math.ucr.edu/home/baez/networks/, 2014.

3. J. C. Baez and J. Erbele. Categories in control. CoRR, abs/1405.6881, 2014. http://arxiv.org/abs/1405.6881.

4. M. Barnabei, C. Guerrini, and L. Montefusco. Some algebraic aspects of signal processing. Linear Algebra and its Applications, 284(1-3):3 - 17, 1998.

5. H. Basold, M. Bonsangue, H. H. Hansen, and J. Rutten. (co)algebraic characterizations of signal flow graphs. In To appear in LNCS, 2014.

6. J. Berstel and C. Reutenauer. Rational series and their languages. EATCS Monographs on Theoretical Computer Science, 12, 1988.

7. F. Bonchi, P. Sobociński, and F. Zanasi. Interacting bialgebras are Frobenius. In FoSSaCS '14, volume 8412 of $L N C S$, pages 351-365. Springer, 2014.

8. F. Bonchi, P. Sobociński, and F. Zanasi. Interacting Hopf algebras. CoRR, abs/1403.7048, 2014. http://arxiv.org/abs/1403.7048.

9. R. Bruni, I. Lanese, and U. Montanari. A basic algebra of stateless connectors. Theor Comput Sci, 366:98-120, 2006.

10. R. Bruni, H. C. Melgratti, U. Montanari, and P. Sobociński. Connector algebras for C/E and P/T nets' interactions. Log Meth Comput Sci, 9(16), 2013.

11. B. Coecke, R. Duncan, A. Kissinger, and Q. Wang. Strong complementarity and non-locality in categorical quantum mechanics. In $\operatorname{LiCS} S^{`} 12$, pages 245-254, 2012.

12. P. Katis, N. Sabadini, and R. F. C. Walters. Span(Graph): an algebra of transition systems. In AMAST '97, pages 322-336. Springer, 1997.

13. S. Lack. Composing PROPs. Theor App Categories, 13(9):147-163, 2004.

14. B. Lahti. Signal Processing and Linear Systems. Oxford University Press, 1998.

15. S. Mac Lane. Categorical algebra. Bull Amer Math Soc, 71:40-106, 1965.

16. S. J. Mason. Feedback Theory: I. Some Properties of Signal Flow Graphs. Massachusetts Institute of Technology, Research Laboratory of Electronics, 1953.

17. S. Milius. A sound and complete calculus for finite stream circuits. In LICS, 2010.

18. J. J. M. M. Rutten. A tutorial on coinductive stream calculus and signal flow graphs. Theor. Comput. Sci., 343(3):443-481, 2005.

19. P. Selinger. Dagger compact closed categories and completely positive maps: (extended abstract). Electr. Notes Theor. Comput. Sci., 170:139-163, 2007.

20. P. Selinger. A survey of graphical languages for monoidal categories. arXiv:0908.3347v1 [math.CT], 2009.

21. P. Sobociński. Representations of Petri net interactions. In CONCUR '10, 2010.

22. J. C. Willems. The behavioural approach to open and interconnected systems. IEEE Contr. Syst. Mag., 27:46-99, 2007. 